\title{
Angiographic derived endothelial shear stress: a new predictor of atherosclerotic disease progression
}

\author{
Christos V. Bourantas, ${ }^{\mathrm{a}, \mathrm{b}, \#{ }^{*}}$ MD, PhD; Anantharaman Ramasamy, ${ }^{\mathrm{a}, \#} \mathrm{MBChB}, \mathrm{MRCP}$; \\ Alexios Karagiannis, ${ }^{\mathrm{c}} \mathrm{PhD}$; Antonis Sakellarios, ${ }^{\mathrm{d}} \mathrm{BSc}$; Thomas Zanchin, ${ }^{\mathrm{e}} \mathrm{MD}$; Kyohei Yamaji, ${ }^{\mathrm{e}} \mathrm{MD}, \mathrm{PhD}$; \\ Yasushi Ueki, ${ }^{\mathrm{e}} \mathrm{MD}$; Xiaohui Shen, ${ }^{\mathrm{f}}$ MSc; Dimitrios I. Fotiadis, ${ }^{\mathrm{d}} \mathrm{PhD}$; Lampros K. Michalis, ${ }^{\mathrm{h}} \mathrm{MD}$, FRCP; \\ Anthony Mathur, ${ }^{a} \mathrm{MD}, \mathrm{PhD}$; Patrick W. Serruys, ${ }^{\mathrm{i}} \mathrm{MD}, \mathrm{PhD}$; Hector M. Garcia-Garcia, ${ }^{\mathrm{j}} \mathrm{MD}, \mathrm{PhD}$; Kostantinos \\ Koskinas, ${ }^{\mathrm{e}} \mathrm{MD}, \mathrm{MSc}$; Ryo Torii, ${ }^{\mathrm{f}} \mathrm{Msc}, \mathrm{PhD}$; Stephan Windecker, ${ }^{\mathrm{e}} \mathrm{MD}$; Lorenz Räber, ${ }^{\mathrm{e}, *} \mathrm{MD}, \mathrm{PhD}$
}

${ }^{\#}$ The first two authors contributed equally to this work

${ }^{a}$ Department of Cardiology, Barts Heart Centre, Barts Health NHS Trust, London, UK

${ }^{\mathrm{b}}$ Institute of Cardiovascular Sciences, University College London, London, UK

${ }^{\mathrm{c}}$ CTU Bern, Institute of Social and Preventive Medicine, Bern University, Bern, Switzerland

${ }^{\mathrm{d}}$ Department of Cardiology, Bern University Hospital, Bern, Switzerland

${ }^{\mathrm{e}}$ Department of Materials Science and Engineering, University of Ioannina, Ioannina, Greece

${ }^{\mathrm{f}}$ Department of Mechanical Engineering, University College London, UK

${ }^{\mathrm{j}}$ The Heart Centre, Rigshospitalet, Copenhagen University Hospital, Copenhagen, Denmark

${ }^{\mathrm{g}} 2^{\text {nd }}$ Department of Cardiology, Medical School, University of Ioannina, Ioannina, Greece

${ }^{\mathrm{h}}$ International Centre for Circulatory Health, NHLI, Imperial College London, London, UK

${ }^{\mathrm{i}}$ Section of Interventional Cardiology, MedStar Washington Hospital Center, Washington, District of Columbia

From the Department of Cardiology, Barts Heart Centre, Barts Health NHS, London, UK

*Address for correspondence

Christos Bourantas, MD, PhD,

Consultant Cardiologist, Barts Heart Centre,

Honorary Senior Lecturer, University College London

The Barts Heart Centre, West Smithfield, London EC1A 7BE

E-mail: cbourantas@gmail.com

Phone: +44 2073777000

Fax: +44 2077919670

Lorenz Räber, MD, $\mathrm{PhD}$

Associate Professor

Bern University Hospital, 3010 Bern, Switzerland

E-mail: Lorenz.Raeber@insel.ch

Phone: +41316324497

Fax: +41 316324770 


\begin{abstract}
Aims: To examine the efficacy of angiography derived endothelial shear stress (ESS) in predicting atherosclerotic disease progression.

Methods and results: Thirty five patients admitted with ST-elevation myocardial infarction that had 3-vessel intravascular ultrasound (IVUS) immediately after revascularization and at 13 months follow-up were included. Three dimensional (3D) reconstruction of the non-culprit vessels were performed using a) quantitative coronary angiography (QCA) and b) methodology involving fusion of IVUS and biplane angiography. In both models, blood flow simulation was performed and the minimum predominant ESS was estimated in 3mm segments. Baseline plaque characteristics and ESS were used to identify predictors of atherosclerotic disease progression defied as plaque area increase and lumen reduction at follow-up.
\end{abstract}

Fifty four vessels were included in the final analysis. A moderate correlation was noted between ESS estimated in the 3D QCA and the IVUS-derived models ( $\mathrm{r}=0.588, \mathrm{P}<0.001) ; 3 \mathrm{D}$ QCA accurately identified segments exposed to low $(<1 \mathrm{~Pa}$ ) ESS in the IVUS-based reconstructions (AUC: 0.793, P<0.001). Low 3D QCA-derived ESS $(<1.75 \mathrm{~Pa})$ was associated with an increase in plaque area, burden and necrotic core at follow up. In multivariate analysis low ESS estimated either in 3D QCA (OR: 2.07, 95\%CI: 1.17-3.67; P=0.012) or in IVUS (<1Pa; OR: 2.23, 95\%CI: 1.23-4.03; $\mathrm{P}=0.008)$ models and plaque burden were independent predictors of atherosclerotic disease progression; 3D QCA and IVUS-derived models had a similar accuracy in predicting disease progression (AUC: 0.826 vs. $0.827, \mathrm{P}=0.907$ ).

Conclusions: 3D QCA-derived ESS can predict disease progression. Further research is required to examine its value in detecting vulnerable plaques.

Keywords: vulnerable plaque, shear stress, intravascular ultrasound. 


\section{Introduction}

Cumulative evidence has shown that an invasive evaluation of coronary plaque morphology can provide useful prognostic information and identify, with however low accuracy, lesions that are likely to progress and cause cardiovascular events.(1-3) This predictive accuracy increases considerably when information about plaque physiology and in particular the endothelial shear stress (ESS) distribution is added to the prognostic model.(46) Traditionally, ESS computation requires the use of tedious and time consuming methodologies that rely on the fusion of intravascular imaging data and coronary angiography and thus these approaches cannot be used in the clinical setting while the patient is on the catheterization laboratory.(7)

On the other hand three dimensional quantitative coronary angiography (3D QCA) can be performed in real time, it appears able to accurately assess luminal dimensions(8) and it has been used to evaluate lumen geometry(9) and detect haemodynamically significant lesions;(10-11) however there is limited evidence about its value in assessing the ESS distribution.(12-13) The aim of this study is to explore whether basic computational fluid dynamic (CFD) processing of 3D QCA models - which can be performed in real time while the patient is on the catheterization laboratory - can reliable evaluate ESS distribution and identify segments that are likely to exhibit atherosclerotic disease progression.

\section{Methods}

\section{Study patients}

We analysed the angiography and intravascular ultrasound (IVUS) imaging data acquired from the non-culprit vessels of patients recruited in the Integrated Biomarkers Imaging 4 (IBIS 4) study (NCT00962416), a substudy of the COMFORTABLE AMI trial. The study protocol has already been described in detail;(14) in summary the study included 103 patients admitted with ST-elevation myocardial infarction who had successful revascularization and serial 3-vessel IVUS, IVUS-virtual histology (VH) and optical coherence tomography imaging at baseline immediately after stent implantation and at 13 months follow-up after receiving intensive treatment with rosuvastatin. 
In the present analysis we included only the patients that had biplane angiography and IVUS imaging at baseline and follow-up. Segments where there was IVUS hang up - that resulted in a length difference $>3 \mathrm{~mm}$ between baseline and follow-up examinations - and those with a short length $(<15 \mathrm{~mm})$ were excluded from the study.

\section{Data acquisition and processing}

\section{IVUS imaging}

IVUS imaging was performed in the culprit and non-culprit vessel using a 20MHz catheter (Eagle Eye, Volcano Cooperation, and San Diego, CA). The catheter was advanced distally to a side branch that was used as anatomical landmark and then was pulled back at a constant speed of $0.5 \mathrm{~mm} / \mathrm{s}$ using an automated pullback device. The pullback was completed when the catheter was back into the left main stem, or the guide catheter in the cases of right coronary artery imaging. All the recruited patients underwent repeat angiography and IVUS imaging at 13 months follow-up. An effort was made to study the same segment based on the most proximal and distal anatomical landmarks in baseline IVUS. Imaging at follow-up was performed to the exact standard as the baseline imaging protocol.

The IVUS data were anonymised and analysed by an independent Core Laboratory (Cardialysis B.V., Rotterdam, The Netherlands) using validated imaging software (QIvus, Medis, Leiden, The Netherlands). The present analysis included only the IVUS data acquired in the non-culprit vessels; in these data side branches were identified and were used to define the segment of interest, i.e., the largest segment that was assessed by IVUS both at baseline and follow-up. For this segment the lumen, external elastic membrane (EEM), the plaque burden and its composition, derived by IVUS-VH (i.e., fibrotic fibrofatty, calcific and necrotic core area and burden) were estimated in every end-diastolic frame (at $\sim 0.4 \mathrm{~mm}$ interval).(14)

\section{Coronary artery reconstruction}

The proximal and distal end of the segment of interest were identified in coronary angiography (Supplementary Figure 1) and then this segment was reconstructed using two methodologies: the first (IVUS-based reconstruction) relies on the fusion of coronary angiography with IVUS and has already been described and validated in detail.(7) This approach enables reliable coronary reconstruction from data acquired during routine 
invasive coronary imaging and accurate evaluation of ESS distribution, and involves the following steps: extraction of the luminal centreline from two angiographic projections, placement of the IVUS contours perpendicularly onto the luminal centreline and estimation of their absolute orientation using side branches that are visible in both angiography and IVUS.(7) This approach was used to reconstruct the segment of interest from the IVUS data acquired both at baseline and follow-up examinations.

The $2^{\text {nd }}$ reconstruction methodology (the $3 D Q C A$ reconstruction) was used to reconstruct the same segment of interest (assessed by IVUS at baseline and follow-up) only at baseline and was performed using well validated software (QAngio XA 3D RE, Medis Specials bv, Leiden, the Netherlands).(15) In brief, two end-diastolic angiographic projections ( $>25^{\circ}$ apart), where there was no overlapping or foreshortening of the segment of interest and enabled accurate delineation of the lumen silhouette were selected; after image calibration its lumen centreline and the lumen borders were automatically identified in the two projections using an established edge detections algorithms. Corresponding points were identified in the two centrelines and used to extract the 3D centreline of the segment of interest. For each pair of corresponding points two lumen diameters were estimated in the two angiographic projections that were used to define an ellipse that corresponded to the lumen cross section in the 3D lumen model.

\section{Blood flow simulation and data processing}

The baseline models were processed with computational fluid dynamic (CFD) techniques using proprietary software (ICEM CFD and CFX 14.5, Ansys, Canonsburg, PA). The same simulation was performed and the same boundary conditions were used in both IVUS- and 3D QCA-based models. Blood was considered homogenous, Newtonian fluid with a dynamic viscosity of $0.0035 \mathrm{~Pa}$ and a density of $1,050 \mathrm{~kg} / \mathrm{m}^{3}$; blood flow was assumed to be steady, laminar and incompressible; the arterial wall was considered to be rigid; no-slip conditions were applied to the lumen surfaces and zero pressure conditions were imposed at the outlet of the model. A flat flow profile was imposed at the inlet of the lumen which it was assumed that was fully developed after the first $3 \mathrm{~mm}$ of the reconstruction. In IVUS-based and QCA-based models blood flow was calculated by measuring the number of frames required for the contrast dye to pass from the inlet to outlet of the segment of interest, the volume of the model and the cine frame rate.(16) 
The baseline IVUS and 3DQCA models were divided in 3mm consecutive segments; the first and last segment of each model were excluded from the analysis and for the remaining $3 \mathrm{~mm}$ segments the minimum predominant ESS values were estimated which, as it has been shown in the PREDICTION study, appear able to predict atherosclerotic disease progression.(5-6) In addition, in the IVUS-based models for each $3 \mathrm{~mm}$ segment the mean lumen, EEM, plaque area, plaque burden and the area and burden of each tissue component as well as the remodelling pattern (i.e., excessive expanding remodelling, positive compensatory and negative remodelling) were computed.(6)

The follow-up IVUS-based models were processed similarly to the IVUS-based reconstructions. The models were divided in $3 \mathrm{~mm}$ segments and for each segment the mean lumen, EEM, plaque area and plaque burden and its composition were estimated.

\section{Statistics}

Continuous values are presented as median and interquartile range and categorical as counts and percentages. Spearman correlation coefficient, linear logistic analysis and Bland and Altman analysis were used to examine the association between models' length and the association between the minimum predominant ESS estimated in the IVUS-based and 3D QCA reconstructions. Receiver-operating characteristics (ROC) curve analysis was used to examine the efficacy of the 3D-QCA derived ESS in detecting segments exposed to low $(<1 \mathrm{~Pa})$ atheropromoting ESS on IVUS-based reconstructions.(6)

To compare plaque features between baseline and follow-up IVUS examinations linear and logistic mixed effects models were used with a random effect at patient level to adjust for the multiple segments per patient. Univariate linear regression models were applied to associate baseline variables with the changes in lumen area, plaque area, plaque burden and necrotic core area. Variables associated with these changes $(\mathrm{P}<0.1)$ were entered into a multivariate model. In case of co-linearity $(r>0.5, \mathrm{P}<0.05)$ only the variable with the higher $\beta$ was entered into the multivariate analysis. All models included a random patient-specific intercept. 
Atherosclerotic disease progression was defined as a reduction in lumen and an increase in plaque area at followup. Binary mixed effects logistic regression analysis was used to identify predictors of disease progression and the variables that appear as independent predictors were used to build two multivariate models: the first included the minimum predominant ESS estimated in IVUS-based models and the other the ESS in the 3D QCA reconstructions. The efficacy of the two models in detecting disease progression was compared using ROC curve analysis.

Analyses were performed in Stata (version 14.2, StataCorp LP, College Station, TX) and R (version 3.4.2, R Foundation for Statistical Computing, Vienna).

\section{Results}

\section{Studied population}

Eighty two (151 vessels) out of the 103 recruited patients had IVUS imaging at baseline and follow-up. From these patients, 50 patients ( 88 vessels) had biplane angiography. After excluding segments with short length, vessels where there was IVUS hang-up during pullback and these where post-processing of the final IVUSbased models were not feasible, fusion of IVUS with X-ray angiography and blood flow simulation was successfully performed in 73 coronary segments. From these 19 segments were excluded from the analysis as accurate 3D QCA reconstruction was not feasible; therefore 35 patients (54 vessels) were included the final analysis (Figure 1). The baseline characteristics of the studied population are illustrated in Table 1.

\section{Association between ESS estimated in IVUS- and QCA-based models}

The median length of the 54 IVUS-based models was $31.71 \mathrm{~mm}(22.47-44.01 \mathrm{~mm})$; after excluding the proximal and distal $3 \mathrm{~mm}$ segments $4703 \mathrm{~mm}$ segments were included in the final analysis. A strong and statistical significant correlation was noted between the length of the 3D QCA (median: 30.95mm, IQR: 23.00-43.00mm) and the IVUS-based models $(\mathrm{r}=0.936, \mathrm{P}<0.001)$. On the other hand, the correlation of the minimum predominant ESS estimated in the IVUS-based reconstructions (median: 1.12Pa, IQR: 0.65-1.80Pa) and 3D QCA models (median: 1.60Pa, IQR: $0.92-2.80 \mathrm{~Pa})$ was moderate but statistical significant $(\mathrm{r}=0.588, \mathrm{P}<0.001$, Figure $2 \mathrm{~A})$. 
Bland-Altman analysis demonstrated a mean difference between IVUS- and 3D QCA-derived ESS of $0.75 \pm 1.85 \mathrm{~Pa}$ indicating that $3 \mathrm{D}$ QCA overestimates the ESS; it is apparent in Figure 2B that the scatter is higher in high ESS compared to low ESS values. ROC curve analysis demonstrated that 3D QCA-derived ESS has a high accuracy in identifying segments exposed to low (<1Pa) ESS in the IVUS-based models (AUC: 0.793). A cutoff of $1.76 \mathrm{~Pa}$ was able to identify segments exposed to low $(<1 \mathrm{~Pa})$ ESS with a moderate specificity and positive predictive value $(0.672$ and 0.656$)$ and a high sensitivity and negative predictive value $(0.810$, and 0.820 respectively; Figure 3A). This cutoff was used to define low ESS in the QCA-based reconstructions and explore its value in predicting atherosclerotic evolution.

\section{Predictors of atherosclerotic disease progression}

At follow-up, both the lumen and plaque area decreased. This should be attributed to the aggressive treatment with rosuvastatin. The reduction in plaque area was due to a decrease in the fibrous and fibroffaty tissue component while the calcific tissue increased at follow-up (Supplementary table 1).

Low minimum predominant ESS in IVUS-based $(<1 \mathrm{~Pa})$ but not in 3D QCA reconstructions $(<1.76 \mathrm{~Pa})$ were associated with an increase in plaque area, plaque burden and necrotic core component at 13 months follow-up. Low IVUS-based ESS was associated with lumen reduction at follow-up while in 3D QCA models the association between low predominant ESS and lumen changes was not statistical significant. In multivariate analysis low predominant ESS in the IVUS-based models was independently associated with the changes in lumen area, plaque area and burden but not with the changes in the necrotic core component, whereas low predominant ESS in the 3D QCA reconstructions was related only with the changes in plaque area (Table 2).

\section{Predictors of atherosclerotic disease progression}

At 13 months follow-up atherosclerotic disease progression was noted in $24 \%(\mathrm{n}=113)$ of the studied $3 \mathrm{~mm}$ segments. The predictors of atherosclerotic disease progression are listed in Table 3. Decreasing baseline plaque burden and low minimum predominant ESS estimated either by IVUS-based or 3D QCA CFD modelling were predictors of disease progression in multivariate analysis. The model that included the ESS estimated in the 
IVUS-based reconstruction and the model that included the ESS derived from the QCA reconstructions had the same accuracy in predicting disease progression (AUC: 0.827 vs. $0.826, \mathrm{P}=0.907$; Figure 3B).

\section{Discussion}

In this study we examined for the first time in a large number of vessels the efficacy of 3D QCA CFD modelling in assessing the local hemodynamic forces and predicting atherosclerotic disease progression. We found: 1) a moderate but significant correlation between the minimum predominant ESS estimated in models reconstructed by 3D QCA and the ESS computed in IVUS-based reconstructions, 2) that 3D QCA is able to identify with high accuracy segments exposed to low ESS in the IVUS-based models $(<1 \mathrm{~Pa}), 3)$ that low minimum predominant ESS values in 3D QCA are associated with the changes in plaque and necrotic core component at follow-up, and 4) that the ESS estimated in 3D QCA and in IVUS-based reconstructions have the same efficacy in predicting atherosclerotic disease progression (Figure 3).

Cumulative evidence has shown that computational modelling can be used to process 3D QCA data and accurately evaluate the hemodynamic significance of coronary artery lesions.(10,17) Advances in software technology have enabled real time assessment of pressure drop in 3D QCA models carrying promise for future applications in the clinical arena.(11) Despite these encouraging results the full potential of 3D QCA in assessing vessel physiology has not been explored yet. Today only two small scale studies that included 7 and 9 coronary arteries have examined the accuracy of the 3D QCA in assessing the ESS distribution and showed a statistical significant correlation between the ESS estimated in 3D QCA and IVUS-based models.(12-13) Moreover, the study of Timmins et al that included serial IVUS imaging at baseline and 6 months follow-up showed that in contrast to IVUS-based reconstructions, the ESS estimated in 3D QCA-models were unable to predict disease progression.(12) However, this analysis had significant limitations as it included only 9 vessels and it used a 3D QCA software which assumed that the lumen has circular cross sections - therefore it was unable to accurately reconstruct eccentric lesions.(12)

The present analysis overcomes the above limitations as it includes a large number of segments and uses a software that is able to generate elliptical cross-sections of the lumen and thus to accurately reconstruct eccentric 
lesions. More importantly this study included only patients who had biplane coronary angiography, we processed angiographic projection that enabled accurate delineation of the lumen borders and we excluded a considerable number of segments (26\%) because of suboptimal angiographic projections. We found a statistical significant - yet moderate - correlation between the ESS estimated in 3D QCA and IVUS-based models but more importantly we demonstrated that 3D QCA has a high accuracy in detecting segments that are exposed to a low (<1 Pa) athero-promoting ESS environment.(6) Low minimum predominant ESS estimated in 3D QCA models appears able to predict changes in plaque and necrotic core dimensions in univariate analysis and was an independent predictor of the changes in plaque area, but, in contrast to IVUS-based ESS, it was not independently associated with the changes in lumen area and plaque burden. The superiority of IVUS-based modelling in assessing ESS distribution and its effect on plaque pathology should be attributed to the fact that IVUS, compared to 3D QCA, enables more accurate reconstruction of lumen geometry. IVUS has a higher axial resolution than QCA and allows detailed visualisation of the lumen border that can have a complex morphology, especially in diseased vessels. Conversely, coronary angiography enables a 2D assessment of the lumen silhouette, while 3D QCA assumes that the lumen has elliptical cross sections that are generated from the lumen diameters measured in corresponding points in QCA and therefore it is likely to provide inaccurate estimation is hazy, complex lesions. However, it appears that in our study these limitations did not affect the predictive value of 3D QCA-derived ESS in detecting segments that will exhibit disease progression at 13 months followup.

The findings of our analysis highlight the potential value of 3D QCA modelling in identifying segments that are exposed in an unfavourable haemodynamic environment and are likely to exhibit disease progression. Consistent evidence has shown that the local haemodynamic forces combined with the plaque characteristics, can detect lesions that are likely to progress and cause events with a positive predictive value that exceeds $>50 \%$ carrying promise for an early detection of vulnerable lesions.(5, 18-19) However, these studies estimated the ESS in models reconstructed form IVUS data and it is well known that this process is laborious and time consuming and thus it cannot be used in the clinical setting. In contrary, 3D QCA can be performed within few minutes, while the patient is on the catheterization laboratory, offering unique opportunities for a real time assessment of vessel physiology. The results of the present analysis provide proofs of the value of 3D QCA 
CFD modelling in the search of the vulnerable plaque; however it has to be acknowledged that this analysis has soft imaging endpoints. Therefore, further research is needed to examine the efficacy of 3D QCA modelling in high-risk plaques and effort should be made to develop user-friendly software that will incorporate computational inexpensive methodologies which will allow fast computation of the ESS distribution before advocating its broad use in detecting high-risk lesions.(20)

\section{Limitations}

A limitation of the present analysis is the fact that both IVUS-based and 3D QCA models did not include the side branches on the reconstructed geometry, which can possibly affect the ESS distribution as shown in a previous report.(21) In addition, we assumed a steady flow profile and thus did not investigate the role of oscillating ESS on plaque progression.(22) These approximations however were made intentionally so as to reduce the computational time and explore the potential of 3D QCA, coupled with basic CFD analysis, in the clinical setting, while the patient is in the catheterization laboratory, to identify and treat vulnerable plaques.(56) In addition, as it has already been stated, although this study in the largest of its kind the number of patients and segments included was too small to allow us to investigate the value of 3D-QCA-based modelling in detecting plaques that progress and caused events. Another limitation of the analysis is the fact that CFD processing of 3D QCA is of value only in cases of optimal angiographic projections. Finally, it has to be stressed that the studied patients received high dose of statins which changed plaque burden and composition at followup and thus it is unclear whether these findings also apply to other populations.

\section{Conclusions}

CFD analysis of 3D QCA models enables evaluation of the local hemodynamic forces and detection with high accuracy of segments that are exposed to an unfavourable hemodynamic environment and are likely to exhibit disease progression at follow-up. Further research is required to examine the value of 3D QCA modelling in detecting high-risk plaques that are likely to progress and cause events. 


\section{Conflict of interest}

None.

\section{Funding}

The IBIS4 trial was supported by the Swiss National Science Foundation and St. Jude Medical. Anantharaman Ramasamy was funded by research funds of Whipps Cross University Hospital, London.

\section{Acknowledgement}

None.

\section{References}

1. Bourantas CV, Garcia-Garcia HM, Torii R, Zhang YJ, Westwood M, Crake T, et al. Vulnerable plaque detection: an unrealistic quest or a feasible objective with a clinical value? Heart. 2016 Apr;102(8):581-9. PubMed PMID: 26783236. Epub 2016/01/20. eng.

2. Stone GW, Maehara A, Lansky AJ, de Bruyne B, Cristea E, Mintz GS, et al. A prospective natural-history study of coronary atherosclerosis. N Engl J Med. 2011 Jan 20;364(3):226-35. PubMed PMID: 21247313. Epub 2011/01/21. eng.

3. Calvert PA, Obaid DR, O'Sullivan M, Shapiro LM, McNab D, Densem CG, et al. Association between IVUS findings and adverse outcomes in patients with coronary artery disease: the VIVA (VH-IVUS in Vulnerable Atherosclerosis) Study. JACC Cardiovasc Imaging. 2011 Aug;4(8):894-901. PubMed PMID: 21835382. Epub 2011/08/13. eng.

4. Thondapu V, Bourantas CV, Foin N, Jang IK, Serruys PW, Barlis P. Biomechanical stress in coronary atherosclerosis: emerging insights from computational modelling. Eur Heart J. 2017 Jan 7;38(2):81-92. PubMed PMID: 28158723. Epub 2017/02/06. eng.

5. Stone PH, Maehara A, Coskun AU, Maynard CC, Zaromitydou M, Siasos G, et al. Role of Low Endothelial Shear Stress and Plaque Characteristics in the Prediction of Nonculprit Major Adverse Cardiac Events: The PROSPECT Study. JACC Cardiovasc Imaging. 2017 Sep 15. PubMed PMID: 28917684. Epub 2017/09/18. eng.

6. Stone PH, Saito S, Takahashi S, Makita Y, Nakamura S, Kawasaki T, et al. Prediction of progression of coronary artery disease and clinical outcomes using vascular profiling of endothelial shear stress and arterial plaque characteristics: the PREDICTION Study. Circulation. 2012 Jul 10;126(2):172-81. PubMed PMID: 22723305. Epub 2012/06/23. eng.

7. Bourantas CV, Papafaklis MI, Athanasiou L, Kalatzis FG, Naka KK, Siogkas PK, et al. A new methodology for accurate 3-dimensional coronary artery reconstruction using routine intravascular ultrasound and angiographic data: implications for widespread assessment of endothelial shear stress in humans. Eurolntervention. 2013 Sep;9(5):582-93. PubMed PMID: 23608530. Epub 2013/04/24. eng.

8. Bourantas CV, Tweddel AC, Papafaklis MI, Karvelis PS, Fotiadis DI, Katsouras CS, et al. Comparison of quantitative coronary angiography with intracoronary ultrasound. Can quantitative coronary angiography accurately estimate the severity of a luminal stenosis? Angiology. 2009 Apr-May;60(2):169-79. PubMed PMID: 18508852. Epub 2008/05/30. eng. 
9. Rittger H, Schertel B, Schmidt M, Justiz J, Brachmann J, Sinha AM. Three-dimensional reconstruction allows accurate quantification and length measurements of coronary artery stenoses. Eurolntervention. 2009 May;5(1):127-32. PubMed PMID: 19577994. Epub 2009/07/07. eng.

10. Papafaklis MI, Muramatsu T, Ishibashi Y, Lakkas LS, Nakatani S, Bourantas CV, et al. Fast virtual functional assessment of intermediate coronary lesions using routine angiographic data and blood flow simulation in humans: comparison with pressure wire - fractional flow reserve. Eurolntervention. 2014 Sep;10(5):574-83. PubMed PMID: 24988003. Epub 2014/07/06. eng.

11. Xu B, Tu S, Qiao S, Qu X, Chen Y, Yang J, et al. Angiography-Based Quantitative Flow Ratio for Online Assessment of Coronary Stenosis: FAVOR II China Study. J Am Coll Cardiol. 2017 Oct 20. PubMed PMID: 29101020. Epub 2017/11/05. eng.

12. Timmins LH, Suo J, Eshtehardi P, Molony DS, McDaniel MC, Oshinski JN, et al. Comparison of angiographic and IVUS derived coronary geometric reconstructions for evaluation of the association of hemodynamics with coronary artery disease progression. Int J Cardiovasc Imaging. 2016 Sep;32(9):1327-36. PubMed PMID: 27229349. Epub 2016/10/19. eng.

13. Toutouzas K, Chatzizisis YS, Riga M, Giannopoulos A, Antoniadis AP, Tu S, et al. Accurate and reproducible reconstruction of coronary arteries and endothelial shear stress calculation using 3D OCT: comparative study to 3D IVUS and 3D QCA. Atherosclerosis. 2015 Jun;240(2):510-9. PubMed PMID: 25932791. Epub 2015/05/02. eng.

14. Raber L, Taniwaki M, Zaugg S, Kelbaek H, Roffi M, Holmvang L, et al. Effect of high-intensity statin therapy on atherosclerosis in non-infarct-related coronary arteries (IBIS-4): a serial intravascular ultrasonography study. Eur Heart J. 2015 Feb 21;36(8):490-500. PubMed PMID: 25182248. Epub 2014/09/04. eng.

15. Tu S, Huang Z, Koning G, Cui K, Reiber JH. A novel three-dimensional quantitative coronary angiography system: In-vivo comparison with intravascular ultrasound for assessing arterial segment length. Catheter Cardiovasc Interv. 2010 Aug 1;76(2):291-8. PubMed PMID: 20665880. Epub 2010/07/29. eng.

16. Bourantas CV, Papafaklis MI, Kotsia A, Farooq V, Muramatsu T, Gomez-Lara J, et al. Effect of the endothelial shear stress patterns on neointimal proliferation following drug-eluting bioresorbable vascular scaffold implantation: an optical coherence tomography study. JACC Cardiovasc Interv. 2014 Mar;7(3):315-24. PubMed PMID: 24529931. Epub 2014/02/18. eng.

17. Tu S, Westra J, Yang J, von Birgelen C, Ferrara A, Pellicano M, et al. Diagnostic Accuracy of Fast Computational Approaches to Derive Fractional Flow Reserve From Diagnostic Coronary Angiography: The International Multicenter FAVOR Pilot Study. JACC Cardiovasc Interv. 2016 Oct 10;9(19):2024-35. PubMed PMID: 27712739. Epub 2016/10/08. eng.

18. Papafaklis MI, Mizuno S, Takahashi S, Coskun AU, Antoniadis AP, Tsuda M, et al. Incremental predictive value of combined endothelial shear stress, plaque necrotic core, and plaque burden for future cardiac events: A post-hoc analysis of the PREDICTION study. Int J Cardiol. 2016 Jan 1;202:64-6. PubMed PMID: 26386924. Epub 2015/09/21. eng.

19. Bourantas CV, Garcia-Garcia HM, Diletti R, Muramatsu T, Serruys PW. Early detection and invasive passivation of future culprit lesions: a future potential or an unrealistic pursuit of chimeras? Am Heart J. 2013 Jun;165(6):869-81 e4. PubMed PMID: 23708157. Epub 2013/05/28. eng.

20. McGregor RH, Szczerbal D, Muralidhar K, Szekely G. A fast alternative to computational fluid dynamics for high quality imaging of blood flow. Med Image Comput Comput Assist Interv. 2009;12(Pt 1):124-31. PubMed PMID: 20425979. Epub 2009/01/01. eng.

21. Sakellarios A, Bourantas CV, Papadopoulou SL, Kitslaar PH, Girasis C, Stone GW, et al. The effect of coronary bifurcation and haemodynamics in prediction of atherosclerotic plaque development: a serial computed tomographic coronary angiographic study. Eurolntervention. 2017 Oct 13;13(9):e1084-e91. PubMed PMID: 28606882. Epub 2017/06/14. eng.

22. Timmins LH, Molony DS, Eshtehardi P, McDaniel MC, Oshinski JN, Giddens DP, et al. Oscillatory wall shear stress is a dominant flow characteristic affecting lesion progression patterns and plaque vulnerability in patients with coronary artery disease. J R Soc Interface. 2017 Feb;14(127). PubMed PMID: 28148771. Pubmed Central PMCID: 5332583. Epub 2017/02/06. eng. 
Table 1. Baseline demographic of the studied patients

\begin{tabular}{|c|c|}
\hline & Studied patients $(n=35)$ \\
\hline Age (years) & $57.2 \pm 10.6$ \\
\hline Gender (male) & $33(94.3 \%)$ \\
\hline Current smoker & $13(37.1 \%)$ \\
\hline \multicolumn{2}{|l|}{ Co-morbidities } \\
\hline Diabetes mellitus & $5(14.3 \%)$ \\
\hline Hypertension & $17(48.6 \%)$ \\
\hline Hypercholesterolaemia & $14(40.0 \%)$ \\
\hline Renal failure $^{a}$ & $1(2.9 \%)$ \\
\hline Previous $\mathrm{PCl}$ & $0(0.0 \%)$ \\
\hline \multicolumn{2}{|l|}{ LV function ${ }^{b}$} \\
\hline Normal LV function & $21(60.0 \%)$ \\
\hline Moderate LV dysfunction & $14(40.0 \%)$ \\
\hline \multicolumn{2}{|l|}{ Clinical presentation } \\
\hline Anterior STEMI & $21(60.0 \%)$ \\
\hline Lateral-posterior STEMI & $3(8.6 \%)$ \\
\hline Inferior STEMI & $11(31.4 \%)$ \\
\hline Studied vessels & $(n=54)$ \\
\hline Left anterior descending artery & $15(27.8 \%)$ \\
\hline Left circumflex coronary artery & $18(33.3 \%)$ \\
\hline Right coronary artery & $21(38.9 \%)$ \\
\hline \multicolumn{2}{|l|}{ Medications at discharge } \\
\hline Aspirin & $35(100 \%)$ \\
\hline Thienopyridines & $35(100 \%)$ \\
\hline Beta-blocker & $33(94.3 \%)$ \\
\hline RAAS inhibitor & $32(91.4 \%)$ \\
\hline Statin & 35 (100\%) \\
\hline
\end{tabular}

LV, left ventricular; PCl, percutaneous coronary intervention; RAAS, renin angiotensin aldosterone system; STEMI, ST-elevation myocardial infarction.

aRenal failure was defined as estimated glomerular filtration rate $<60 \mathrm{~mL} / \mathrm{min} / 1.73 \mathrm{~m} 2$.

${ }^{b}$ Normal LV systolic function: LV ejection fraction >_50\%, moderate LV systolic dysfunction: LV ejection fraction $>30 \%$ and $<50 \%$. 
Table 2. Univariate and multivariate analyses of the variables associated with the changes in lumen dimensions and plaque burden

\begin{tabular}{|c|c|c|c|c|c|c|}
\hline \multirow[b]{2}{*}{ Associate factor } & \multicolumn{2}{|l|}{ Univariate analysis } & \multicolumn{2}{|c|}{$\begin{array}{l}\text { Multivariate model including } \\
\text { the IVUS-based ESS }\end{array}$} & \multicolumn{2}{|c|}{$\begin{array}{l}\text { Multivariate model including } \\
\text { the 3D QCA-based ESS }\end{array}$} \\
\hline & $\beta(95 \% \mathrm{Cl})$ & $\mathbf{P}$ & $\beta(95 \% \mathrm{Cl})$ & $\mathbf{P}$ & $\beta(95 \% \mathrm{Cl})$ & $\mathbf{P}$ \\
\hline \multicolumn{7}{|l|}{ Reduction in lumen area (per $1 \mathrm{~mm}^{2}$ ) } \\
\hline Presence of low predominant ESS in IVUS models & $0.78(0.54-1.02)$ & $<0.001$ & $0.67(0.42-0.93)$ & $<0.001$ & & \\
\hline Presence of low predominant ESS in 3D QCA models & $0.21(-0.03$ to 0.46$)$ & 0.089 & & & $0.12(-0.13-0.37)$ & 0.337 \\
\hline Increasing baseline lumen area (per $1 \mathrm{~mm}^{2}$ ) & $0.12(0.09-0.16)$ & $<0.001$ & & & & \\
\hline Increasing baseline plaque burden (per 1\%) & $-0.21(-0.31$ to -0.10$)$ & $<0.001$ & $-0.11(-0.22$ to -0.00$)$ & 0.045 & $-0.17(-0.28$ to -0.06$)$ & 0.002 \\
\hline Presence of excessive expanding remodelling & $0.45(0.14-0.76)$ & 0.004 & $0.28(-0.03$ to 0.58$)$ & 0.075 & $0.37(0.06-0.68)$ & 0.020 \\
\hline Increasing baseline fibrotic tissue burden (per 1\%) & $0.10(0.00-0.19)$ & 0.044 & & & & \\
\hline \multicolumn{7}{|l|}{ Increase in plaque area (per $1 \mathrm{~mm}^{2}$ ) } \\
\hline Presence of low predominant ESS in IVUS models & $0.67(0.45-0.89)$ & $<0.001$ & $0.27(0.03-0.52)$ & 0.029 & & \\
\hline Presence of low predominant ESS in 3D QCA models & $0.48(0.26-0.70)$ & $<0.001$ & & & $0.24(0.02-0.47)$ & 0.034 \\
\hline Increasing baseline lumen area (per $1 \mathrm{~mm}^{2}$ ) & $0.06(0.02-0.09)$ & 0.005 & & & & \\
\hline Increasing baseline lumen area (per $1 \mathrm{~mm}^{2}$ ) & $-0.15(-0.19$ to -0.12$)$ & $<0.001$ & & & & \\
\hline Increasing baseline plaque burden (per 1\%) & $-0.42(-0.52$ to -0.33$)$ & $<0.001$ & $-0.38(-0.51$ to -0.26$)$ & $<0.001$ & $-0.40(-0.52$ to -0.29$)$ & $<0.001$ \\
\hline Increasing baseline fibrotic tissue burden (per 1\%) & $-0.19(-0.28$ to -0.09$)$ & $<0.001$ & & & & \\
\hline Increasing baseline fibrofatty tissue burden (per 1\%) & $-0.43(-0.70$ to -0.15$)$ & 0.003 & $-0.07(-0.38$ to 0.24$)$ & 0.650 & $-0.04(-0.35$ to 0.26$)$ & 0.778 \\
\hline Increasing baseline necrotic core burden (per 1\%) & $-0.34(-0.48$ to -0.19$)$ & $<0.001$ & & & & \\
\hline Increasing baseline calcific tissue burden (per 1\%) & $-0.25(-0.50$ to -0.00$)$ & 0.047 & $0.08(-0.19$ to 0.36$)$ & 0.558 & $0.13(-0.15$ to 0.40$)$ & 0.366 \\
\hline \multicolumn{7}{|l|}{ Increase in plaque burden (per 1\%) } \\
\hline Presence of low predominant ESS in IVUS models & $0.39(0.28-0.51)$ & $<0.001$ & $0.30(0.18-0.42)$ & $<0.001$ & & \\
\hline Presence of low predominant ESS in 3D QCA models & $0.17(0.05-0.29)$ & 0.007 & & & $0.08(-0.04$ to 0.20$)$ & 0.190 \\
\hline Increasing baseline lumen area (per $1 \mathrm{~mm}^{2}$ ) & $0.05(0.03-0.07)$ & $<0.001$ & & & & \\
\hline Increasing baseline plaque area (per $1 \mathrm{~mm}^{2}$ ) & $-0.03(-0.05$ to -0.01$)$ & 0.009 & & & & \\
\hline Increasing baseline plaque burden (per 1\%) & $-0.17(-0.22$ to -0.12$)$ & $<0.001$ & $-0.13(-0.18$ to -0.07$)$ & $<0.001$ & $-0.16(-0.21$ to -0.10$)$ & $<0.001$ \\
\hline Presence of excessive expanding remodelling & $0.17(0.02-0.32)$ & 0.029 & $0.06(-0.09$ to 0.20$)$ & 0.454 & $0.10(-0.05$ to 0.25$)$ & 0.186 \\
\hline \multicolumn{7}{|l|}{ Increase in necrotic core area (per $1 \mathrm{~mm}^{2}$ ) } \\
\hline Presence of low predominant ESS in IVUS models & $0.13(0.02-0.24)$ & & $0.09(-0.04$ to 0.21$)$ & 0.165 & & \\
\hline Presence of low predominant ESS in 3D QCA models & $0.14(0.03-0.25)$ & & & & $0.08(-0.03$ to 0.20$)$ & 0.157 \\
\hline Increasing baseline lumen area (per $1 \mathrm{~mm}^{2}$ ) & $0.01(-0.00$ to 0.03$)$ & & & & & \\
\hline Increasing baseline plaque burden per (1\%) & $-0.05(-0.10$ to -0.00$)$ & & $-0.03(-0.09$ to 0.03$)$ & 0.304 & $-0.04(-0.10$ to 0.02$)$ & 0.207 \\
\hline Increasing baseline fibrotic tissue burden (per 1\%) & $0.08(0.03-0.12)$ & & & & & \\
\hline Increasing baseline fibrofatty tissue burden (per 1\%) & $0.39(0.26-0.53)$ & $<0.001$ & $0.36(0.21-0.51)$ & $<0.001$ & $0.37(0.22-0.51)$ & $<0.001$ \\
\hline Increasing baseline necrotic core burden (per 1\%) & $0.30(-0.37$ to -0.23$)$ & $<0.001$ & & & & \\
\hline Increasing baseline calcific burden (per 1\%) & $-0.37(-0.49$ to -0.25$)$ & $<0.001$ & $-0.26(-0.40$ to -0.13$)$ & $<0.001$ & $-0.25(-0.39$ to -0.12$)$ & $<0.001$ \\
\hline
\end{tabular}


Table 3. Univariate and multivariate analyses of the factors associated with atherosclerotic disease progression

\begin{tabular}{|c|c|c|c|c|c|c|}
\hline \multirow[b]{2}{*}{ Associate factor } & \multicolumn{2}{|l|}{ Univariate analysis } & \multicolumn{2}{|c|}{$\begin{array}{l}\text { Multivariate analysis } \\
\text { including the IVUS-based ESS }\end{array}$} & \multicolumn{2}{|c|}{$\begin{array}{l}\text { Multivariate analysis including } \\
\text { the 3D QCA-based ESS }\end{array}$} \\
\hline & OR $(95 \% \mathrm{CI})$ & $\mathbf{P}$ & OR $(95 \% \mathrm{Cl})$ & $\mathbf{P}$ & OR $(95 \% \mathrm{Cl})$ & $\mathbf{P}$ \\
\hline Presence of low predominant ESS in IVUS models & $3.01(1.79-5.07)$ & $<0.001$ & $2.07(1.17-3.67)$ & 0.012 & & \\
\hline Presence of low predominant ESS in 3D QCA models & $2.70(1.59-4.58)$ & 0.001 & & & $2.23(1.23-4.03)$ & 0.008 \\
\hline Baseline lumen area (per $1 \mathrm{~mm}^{2}$ increase) & $1.15(1.04-1.28)$ & 0.006 & $1.05(0.94-1.17)$ & 0.814 & $1.02(0.91-1.14)$ & \\
\hline Baseline plaque area (per $1 \mathrm{~mm}^{2}$ increase) & $0.89(0.81-0.98)$ & 0.014 & & & $-0.17(-0.28$ to -0.06$)$ & 0.732 \\
\hline Baseline plaque burden (per $1 \mathrm{~mm}^{2}$ increase) & $0.58(0.45-0.75)$ & $<0.001$ & $0.64(0.49-0.84)$ & 0.001 & $0.65(0.50-0.86)$ & 0.002 \\
\hline Baseline necrotic core burden (per $1 \mathrm{~mm}^{2}$ increase) & $0.65(0.45-0.93)$ & 0.019 & & & & \\
\hline
\end{tabular}

82 patients ( 152 vessels)

had serial IVUS imaging

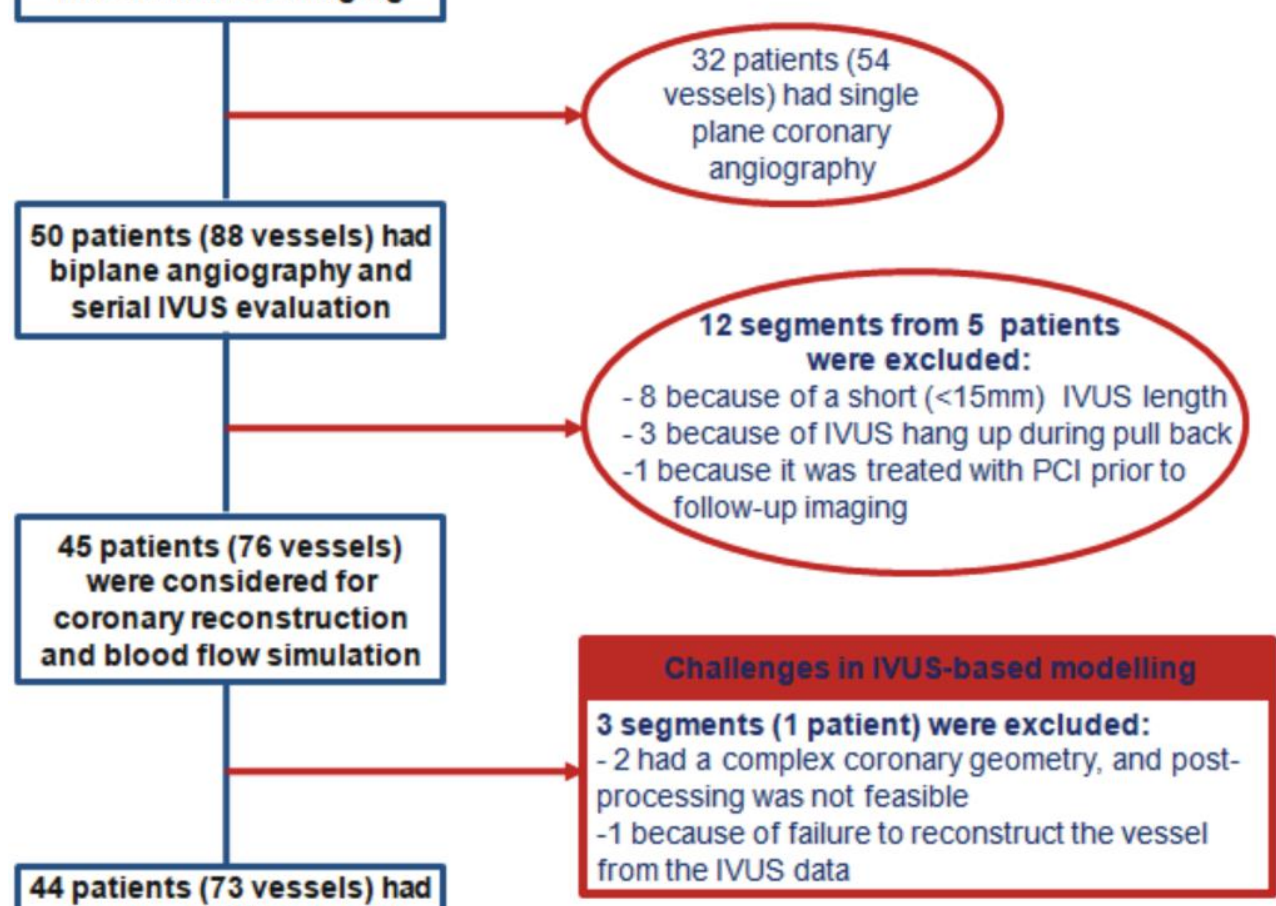

successful IVUS-based

reconstruction and blood

flow simulation

\section{Challenges in 3D QCA modelling}

19 segments from 9 patients were excluded:

-14 because of unsuitable $X$-ay images*

-4 because of absent of information about pixel

size in the DICOM files

-1 because of QCA software failure in

Data from 35 patients ( 54

vessels) were included the

final analysis

*6 segments were excluded because of foreshortening, 7 because of extensive vessel

overlapping, and 1 because of a moderate delineation of the lumen borders in the spider view.

Figure 1. Flowchart of study design. 

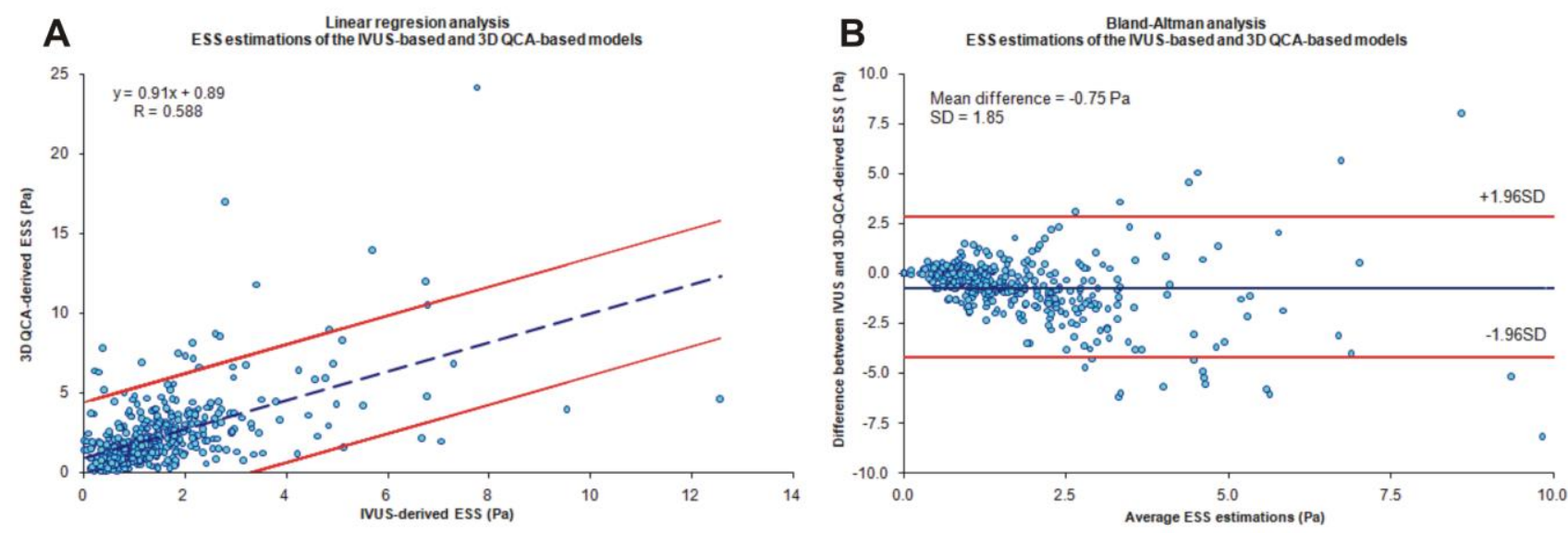

Figure 2. Linear regression analysis (A) and Bland and Altman analysis (B) of the IVUS-based $v s$ the 3D-QCA derived minimum predominant ESS values estimated in the $4703 \mathrm{~mm}$ segments included in the present analysis.
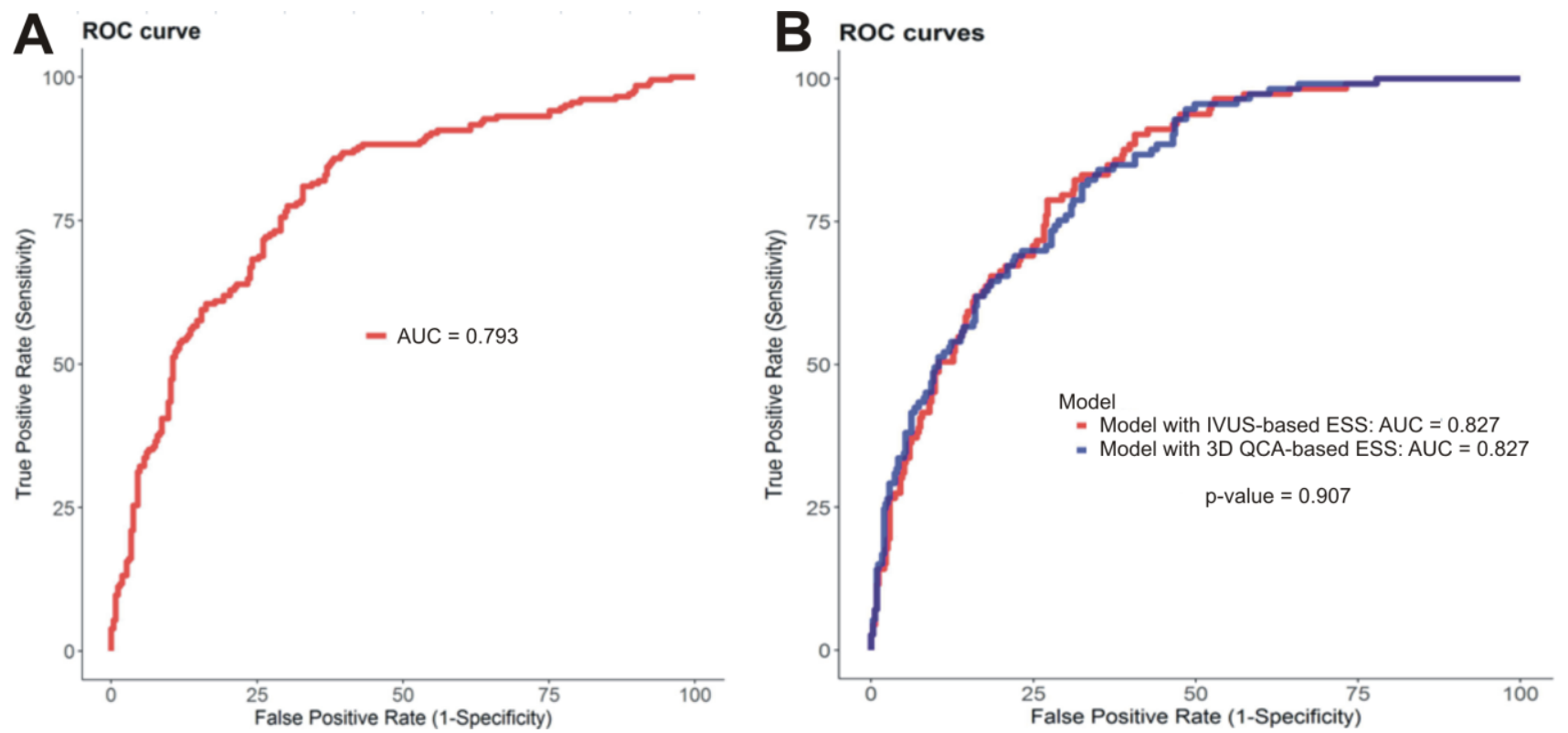

Figure 3. (A) ROC curve plot demonstrating the efficacy of the minimum predominant ESS estimated 3D QCA models in detecting segments that are exposed to low $(<1 \mathrm{~Pa})$ ESS in the models reconstructed from the fusion of IVUS and Xray angiography. A cutoff value of $1.76 \mathrm{~Pa}$ was able to indentify segments exposed to low ESS in IVUS-based reconstructions with an AUC of 0.793. (B) ROC curve analysis demonstrating the efficiency of the model that included the minimum predominant ESS estimated in the 3D QCA (AUC: 0.826) and the model that included the ESS derived from the IVUS-based reconstructions in detecting atherosclerotic disease progression (AUC: 0.827). It is apparent that there is no significant difference in the predictive accuracy of the two models ( $\mathrm{P}=0.907)$. 


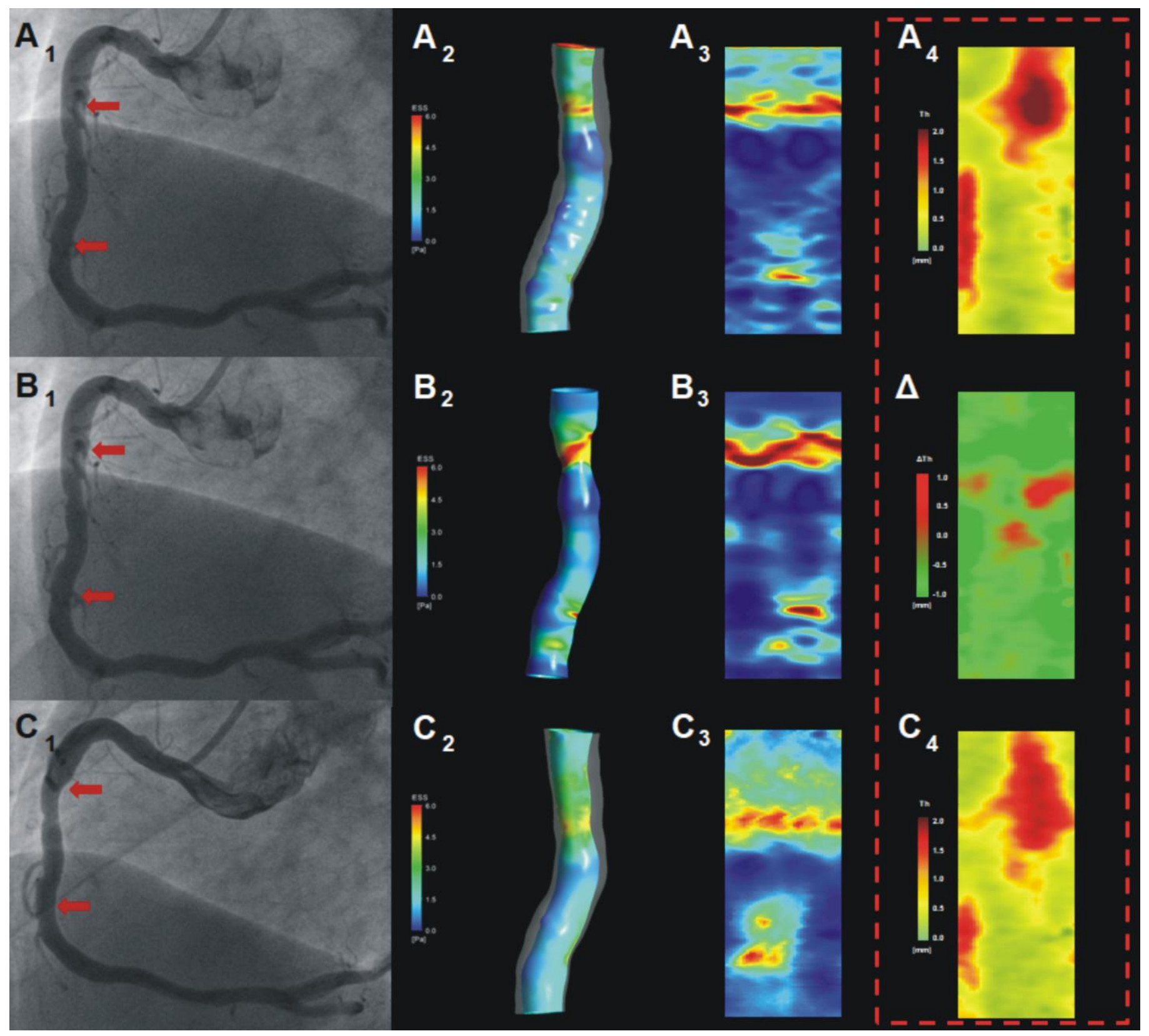

Figure 4. ESS and plaque distribution in a RCA at baseline and 13 months follow-up. $\left(\mathrm{A}_{1}, \mathrm{~B}_{1}\right) \mathrm{X}$-ray angiography of the segments of interest at baseline - its proximal and distal end are indicated with arrows. Panel $\mathrm{A}_{2}$ portrays the segment of interest reconstructed from the fusion of X-ray angiography and IVUS. The ESS distribution in the 3D model is colour coded displayed (blue colour indicates low ESS and red high ESS), while the EEM model in shown in a semitransparent fashion. Panels $\mathrm{A}_{3}$ and $\mathrm{A}_{4}$ portray spread-out plots of the ESS and plaque distribution respectively. The corresponding 3D QCA model and a spread-out plot of the ESS distribution are shown in panels $\mathrm{B}_{2}$ and $\mathrm{B}_{3}$. It is apparent that there is a similarity in ESS values between the IVUS-based and 3D QCA model. Panel $\mathrm{C}_{1}$ shows an angiographic image of the RCA at follow-up. Although lumen's silhouette is similar at baseline and follow-up in coronary angiography, the reconstruction of the segment of interest from the IVUS and X-ray data $\left(\mathrm{C}_{2}, \mathrm{C}_{4}\right)$ shows significant changes in the lumen dimensions and plaque burden $\left(\mathrm{C}_{4}\right)$. These changes have an impact on the ESS distribution at follow-up $\left(\mathrm{C}_{3}\right)$. Panel $\Delta$ shows the changes in plaque thickness between baseline and follow-up (green indicates plaque regression and red plaque progression). Segments exposed to low minimum predominant ESS in the baseline reconstructions (IVUS- or 3D QCA-based) exhibit disease progression at follow-up. 